\title{
Diseño y desarrollo de un entorno virtual inmersivo para instruir el principio de superposición de movimientos a estudiantes de ingeniería
}

\author{
David Castro-González, Luis Hernando Barbosa, Vladimir Prada-Jiménez \& Gregory Conde-Méndez \\ Facultad de Ingeniería y Ciencias Básicas, Universidad Central, Bogotá, Colombia. \\ dcastrog@ucentral.edu.co,lbarbosab@ucentral.edu.co,vpradaj@ucentral.edu.co,gcondem@ucentral.edu.co
}

\begin{abstract}
Resumen-Este artículo describe el diseño y construcción de un entorno de realidad virtual inmersiva con una secuencia de escenas interactivas que recrean la composición de movimientos de una partícula para instruir el principio de superposición de movimientos en estudiantes de Física en ingeniería. El entorno virtual se ha desarrollado en Unity y permite la interacción por medio de las manos con visualización a través de un Head Mounted Display. Este artículo muestra la etapa de diseño, la programación y las principales características del entorno inmersivo logrado. Para probar el entorno inmersivo final se ha interactuado con una población de 20 estudiantes usando un test de 9 preguntas antes y después del uso del entorno inmersivo en cada estudiante. La ganancia normalizada que produce su uso en el grupo de estudiantes, sin acompañamiento y sin interacción social, es $\langle\mathrm{g}\rangle=0.08$.
\end{abstract}

Palabras Clave - entorno inmersivo, realidad virtual, movimiento de partículas, Unity.

Recibido: 6 de diciembre de 2016. Revisado: 17 de enero de 2017. Aceptado: 27 de enero de 2017.

\section{Design and development of an immersive virtual environment for teaching of the superposition of movements principle for engineering students}

Abstract - This article relates the design and construction of an immersive virtual reality environment with a sequence of interactive scenes. These scenes simulate different movements of a particle for the teaching of the superposition principle. The virtual environment has been developed in Unity and the interaction participants are by the fingers and hands with visualization in a Head Mounted Display. To test the final immersive environment we experiment with 20 students. The participants' knowledge acquisition was evaluated by comparing pre-test and post-test. The use of the immersive environment in the group of students, without accompaniment of the teacher and without social interaction between students produced a normalized gain of $\langle\mathrm{g}\rangle=0.08$. Unity.

Keywords - Immersive environment, virtual reality, particle movement,

\section{Introducción}

La dificultad en la enseñanza y aprendizaje de la Física en los primeros semestres de las carreras de ingeniería ha despertado el interés de los profesores de la universidad Central. Uno de los conceptos claves de la Física mecánica que presenta dificultad para ser apropiado por los estudiantes es el principio de superposición de movimientos (PSM). Este principio implica la habilidad de componer movimientos de partículas y constituye una competencia cognitiva relevante para poblaciones de estudiantes de ingeniería. El PSM es una herramienta conceptual que sirve para describir el movimiento de partículas en $2 \mathrm{D}$ y $3 \mathrm{D}$, y según estudios realizados por la asociación americana de profesores de Física, este principio no logra aprenderse cuando se enseña usando la metodología tradicional [1]. En esta misma dirección, en España, hacen un estudio de detección de ideas previas en estudiantes que le da reconocimiento a la enseñanza de este principio [2].

Dice Barbosa y Organista que para lograr que los estudiantes apropien el PSM no es suficiente que el profesor enuncie el postulado, se necesita que tengan mucha experiencia de hechos para que puedan anclar su conocimiento y debido a que el movimiento de objetos lanzados al aire sucede tan rápidamente, el estudiante no tiene suficiente experiencia para anclar y construir este concepto [3]. Para que los estudiantes ganaran experiencia ellos realizaron una secuencia didáctica basada en videos en cámara lenta, con el fin de observar detalladamente diferentes situaciones de movimiento de una partícula y generar experiencia. Sin embargo, ellos reportan baja ganancia de los estudiantes en el aprendizaje de este concepto [3].

Para aportar en esta problemática surgen nuevas herramientas tecnológicas aplicables al proceso de enseñanza/aprendizaje. La realidad virtual (RV) es una de ellas, y consiste en la creación de entornos imaginarios simulados por computadora que se asemejan al mundo real $[4,5]$. La RV puede clasificarse dependiendo del grado de interacción y de inmersión que proveen al usuario, y según [6,7], existen tres clases que son la RV no inmersiva, RV semi-inmersiva y RV de inmersión total.

La RV no inmersiva consiste en una computadora capaz de reproducir contenidos multimedia, permitiendo interactuar a través del teclado, el ratón o palancas hápticas. La RV semiinmersiva proporciona una ligera sensación de inmersión, comúnmente a través de pantallas estereoscópicas. La RV de inmersión total es la mejor opción para transmitir información multi-sensorial porque permite sumergir completamente al

Como citar este artículo: Castro-González, D., Barbosa, L. H., Prada-Jiménez, V. \& Conde-Méndez, G.. Diseño y desarrollo de un entorno virtual inmersivo para instruir el principio de superposición de movimientos a estudiantes de ingeniería. Revista Educación en Ingeniería 12 (23) 101-108, Febrero, 2017. 
usuario en un ambiente virtual (AV) [8], dando una sensación de que se ha "entrado" en un mundo nuevo. Para conseguir dicha inmersión, son necesarios dispositivos de visualización como gafas de RV, software para la creación de mundos tridimensionales y otros accesorios como dispositivos hápticos y sensores de movimiento.

Se han realizado desarrollos para aprendizaje del Inglés como en [9], el aprendizaje de las Matemáticas [10-14], la Química [15,16], y la Historia [17,18]. También se han desarrollado laboratorios remotos virtuales en Automática y Robótica [19] y manipulación de maquinaria [20]. Asimismo en Medicina [21], [22] y Astronomía [23]. En cuanto a la Física la literatura reporta investigaciones para el aprendizaje de los conceptos básicos del electromagnetismo [24], propiedades de los gases [25] y movimiento relativo [26]. Aún no se han desarrollado entornos inmersivos de RV para la enseñanza del PSM.

El propósito de este documento es mostrar el proceso de diseño y construcción de un entorno virtual inmersivo para el aprendizaje del PSM medido con la ganancia normalizada de Hake y los posibles beneficios de esta tecnología como herramienta de acompañamiento en el proceso de enseñanza/aprendizaje. El artículo está estructurado de la siguiente manera: en la sección 2 se describe la metodología, que a su vez, está dividida en sub-secciones: en la sección 2.1 se da una visión general del entorno propuesto, en la sección 2.2 se enlistan las especificaciones de diseño. La sección 2.3 contiene los requisitos de hardware y software del sistema inmersivo. La sección 2.4 presenta la secuencia de escenas. La sección 2.5 muestra el desarrollo del sistema inmersivo. En la sección 3 está el análisis de resultados del entorno inmersivo 3D al implementarlo en una muestra piloto de estudiantes. Finalmente en la sección 4 son presentadas las conclusiones.

\section{Metodología}

\subsection{Visión general del entorno propuesto}

Con el fin de ofrecer a las comunidades de aprendizaje de la Física nuevas herramientas que aporten significativamente en su desempeño académico y fortalezcan su aprendizaje en el aula de clases, este trabajo tuvo como objetivo diseñar y ensamblar un entorno de aprendizaje inmersivo que permitiera a los estudiantes interactuar con un mundo virtual a partir de una secuencia de escenas que simulan diferentes movimientos de una partícula para favorecer el aprendizaje del PSM. Los estudiantes interactúan con objetos propios del entorno inmersivo dentro de un ambiente de aprendizaje centrado en el estudiante.

\subsection{Viabilidad del entorno propuesto}

Los programas de simulación ofrecen gran variedad de herramientas que permiten recrear casi cualquier situación que ocurre en la vida real con gran precisión pudiendo ser ajustadas a conveniencia según sea la necesidad. Para este proyecto se ha utilizado el software de descarga gratuita Unity por su versatilidad para la creación de entornos virtuales. Este programa ha sido seleccionado porque posee un motor de Física integrado que proporciona componentes que manejan la simulación de eventos físicos muy reales con solo ajustar unos cuantos parámetros con instrucciones de código.

En cuanto a recurso humano y financiero, la Universidad Central apoya proyectos de investigación que buscan fortalecer e innovar en el sector académico y tecnológico. Este proyecto ganó el patrocinio en la cuarta convocatoria interna, de la universidad en el que participaron 4 docentes de tiempo completo y un estudiante de pregrado del programa de ingeniería electrónica. Los costos en equipos y dispositivos fue de siete millones de pesos aproximadamente, que incluye un computador de alto rendimiento, un casco de realidad virtual y un sensor de manos. El software de desarrollo es de descarga gratuita, pero si se implementa en varios equipos eleva el costo del estudio.

\subsection{Directrices de diseño del entorno inmersivo}

Se destacan dos características esenciales que debe tener el ambiente virtual propuesto: la primera, debe permitir al estudiante interactuar constantemente con el entorno, y la segunda, debe dar el mayor grado de inmersión posible. En consecuencia, se precisan las siguientes especificaciones a tener en cuenta: 1) Permitir al usuario interactuar con el movimiento del cuerpo. 2) Contar con un menú de opciones: repetir el movimiento, pausar el movimiento, regresar y continuar de escena y ejecutar movimiento. 3) Presentar información gráfica sobre el comportamiento del movimiento de la partícula en tiempo real a partir de gráficas de posición, velocidad y aceleración. 4) Abarcar los conceptos asociados a: movimiento uniforme rectilíneo (mur), movimiento uniformemente acelerado (mua), suma y resta de movimientos (superposición y descomposición). 5) Seguir una secuencia lógica. 6) Hacer síntesis de cada escenario. 7) Tener un cronómetro para dar cuenta del tiempo. 8) Dar instrucciones por medio de grabaciones de audio. 9) Tener marcas de posición para dar cuenta del movimiento de la partícula. 10) Sonidos del ambiente. 11) Ser llamativo y realista. 12) Estimular la vista y el oído. Y finalmente, 13) Tiempo estimado total entre 10 y 20 minutos.

\subsection{Requisitos de hardware y software}

En esta sección se describen los diferentes componentes utilizados en el desarrollo del sistema inmersivo propuesto: dispositivos, equipos y software.

\subsubsection{Dispositivos y equipos}

Se usó un computador de escritorio con un procesador Intel core i7-4790 CPU 3.6 GHz, 16 GB DDR-4 de memoria RAM y una tarjeta de video NVIDIA GeForce GTX-970.

Un kit de desarrollo Oculus Rift DK2 que contiene unas gafas de realidad virtual con una resolución de 960x1080 por ojo, un campo de visión de $100^{\circ}$. Permite seguimiento de la cabeza del usuario y su posición. Un control Leap Motion capaz de captar el movimiento de las manos y los dedos. Este periférico USB se monta sobre las gafas de RV. También se utilizan unos audífonos diadema. En la Fig. 1 se muestra el diagrama de los equipos y dispositivos mencionados anteriormente. 


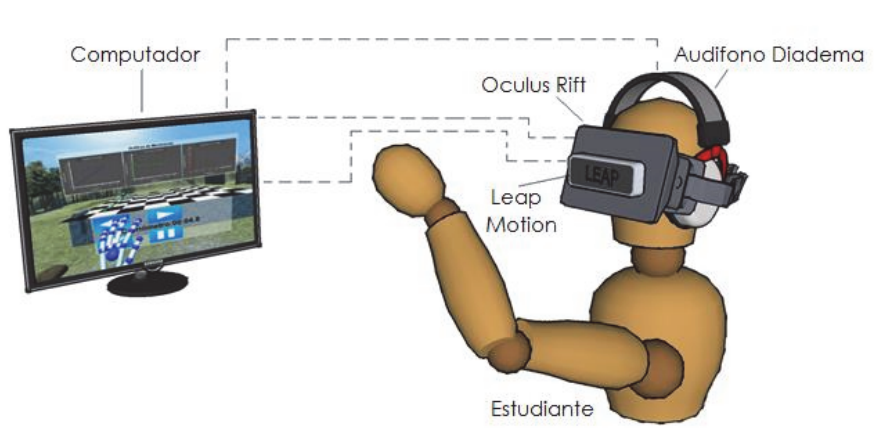

Figura 1. Diagrama de los equipos y dispositivos utilizados en el entorno inmersivo.

Fuente: Los autores.

La visualización del entorno virtual se hace a través del Oculus Rift, el sensor de manos Leap Motion va montado sobre las gafas y los audífonos diadema se ubican sobre los oídos. El Oculus Rift se conecta directamente al computador mediante un cable HDMI y un cable USB 3.0. El Leap Motion y los audiófonos diadema por USB 2.0.

\subsubsection{Software}

Los componentes del software del sistema inmersivo se dividen de la siguiente forma: a) Software: Unity, b) integración de los dispositivos en Unity. Todos los módulos están en función de una sola aplicación e incorporan lo necesario para crear el ambiente virtual inmersivo, tal como se muestra en la Fig. 2.

a) Unity es una de las plataforma más robustas para la creación de videojuegos multiplataforma, además cuenta con soporte para la integración de diversos dispositivos y programas de diseño 3D como 3ds Max, Blender, Cinema 4D, entre otros.

b) Las características adicionales del entorno se consiguen mediante paquetes de descarga gratuita a través del Assets Store (tienda On-line de Unity) como texturas, materiales para objetos, modelos 3D, cielos, entre otros.

La programación del entorno se realiza mediante el entorno de desarrollo integrado de Unity (MonoDevelop) y se integran con las librerías del hardware (Oculus y Leap Motion) y la librería de las gráficas. En cuanto a la parte gráfica, algunos objetos se descargan gratuitamente, otros son creados en Unity.

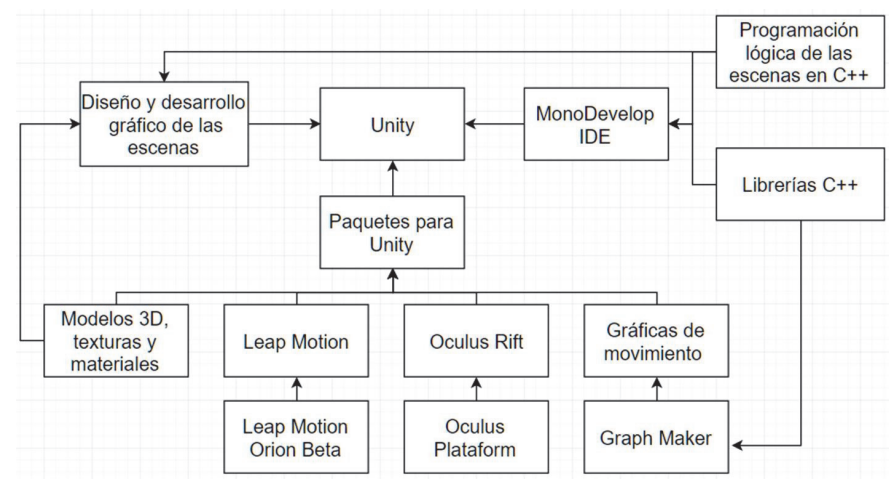

Figura 2. Interacción de los módulos de software. Fuente: Los autores.
Tabla 1

Diseño de actividades de aprendizaje

\begin{tabular}{|c|c|c|}
\hline Tema & Nombre & $\begin{array}{l}\text { Objetivo de } \\
\text { aprendizaje }\end{array}$ \\
\hline $\begin{array}{l}\text { Movimiento } \\
\text { rectilíneo } \\
\text { uniforme y } \\
\text { superposición. }\end{array}$ & $\begin{array}{l}\text { Escena } 1: \text { m.u.r eje } x \\
\text { Escena 2: m.u.r eje y } \\
\text { Escena } 3: \text { m.u.r eje x,y } \\
\text { Escena } 4 \text { : variación de } 3\end{array}$ & $\begin{array}{c}\text { Observar la } \\
\text { trayectoria rectilínea } \\
\text { de una partícula } \\
\text { (esfera) al ser } \\
\text { impulsada en } \\
\text { diferentes direcciones. }\end{array}$ \\
\hline $\begin{array}{l}\text { Movimiento } \\
\text { rectilíneo } \\
\text { uniforme, } \\
\text { movimiento } \\
\text { uniforme } \\
\text { acelerado y } \\
\text { superposición. }\end{array}$ & $\begin{array}{l}\text { Escena 5: m.u.r plano } \\
\text { inclinado. } \\
\text { Escena 6: m.u.r eje y plano } \\
\text { inclinado. } \\
\text { Escena 7: superposición de } 5 \\
\text { y } 6 \text {. } \\
\text { Escena 8: variación de } 7\end{array}$ & $\begin{array}{l}\text { Observar cómo se } \\
\text { genera un movimiento } \\
\text { parabólico a partir de } \\
\text { un mur y un mua en } \\
\text { un plano inclinado. }\end{array}$ \\
\hline
\end{tabular}

Fuente: Los autores.

\subsection{Diseño de la secuencia de escenas}

Para dar cumplimiento a las especificaciones 4 y 5 de la sección 2.2, y teniendo en cuenta el plan de estudios del curso de Física I de la universidad, en la Tabla 1 se resumen la temática, en la cual aparece el nombre y el objetivo de aprendizaje de cada una de las escenas propuestas. Con el fin de generar experiencia en el estudiante sobre situaciones que lleven a componer y descomponer movimientos de partículas se planearon las escenas de la Tabla 1 y de la Fig. 3.

Donde m.u.r (mur) corresponde a movimiento uniforme rectilíneo y m.u.a. (mua) corresponde a movimiento uniformemente acelerado. Estos dos movimientos de partículas se consideran los más simples posibles y si se superponen desde cada componente se puede obtener un movimiento en el plano que puede ser semi-parabólico o parabólico. Nótese que cuando se superpone un mur en una dimensión con un mur en la otra dimensión perpendicular se origina un mur en el plano, pero si se combina un mur con un mua puede surgir una trayectoria semi-parabólica o parabólica del movimiento de la esfera en el plano.

\subsubsection{Escenas para generar experiencia del PSM}

En las escenas de la 1 a la 8, se dispone de una superficie, una esfera en reposo y un taco que interactúa con ella para generar
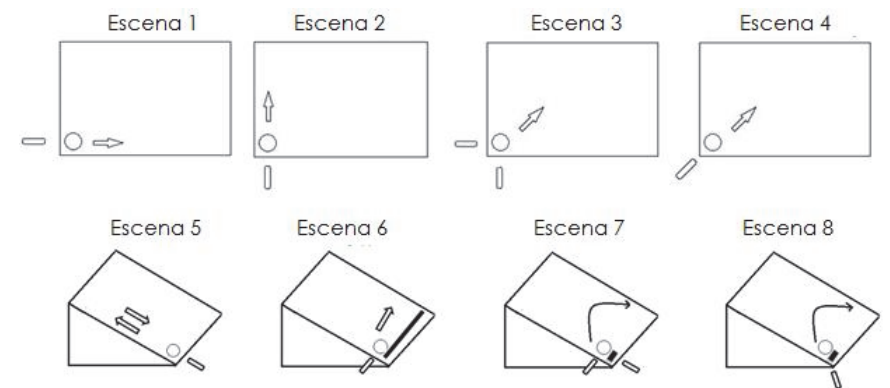

Figura 3. Descripción gráfica de las escenas establecidas por un grupo de expertos en Física que pueden promover el aprendizaje del PSM. Las escenas de la parte superior (1-4) presentan situaciones de movimientos uniformes rectilíneos. Las escenas de la parte inferior (5-8) presentan situaciones de movimientos uniformes acelerados y rectilíneos Fuente: Los autores. 


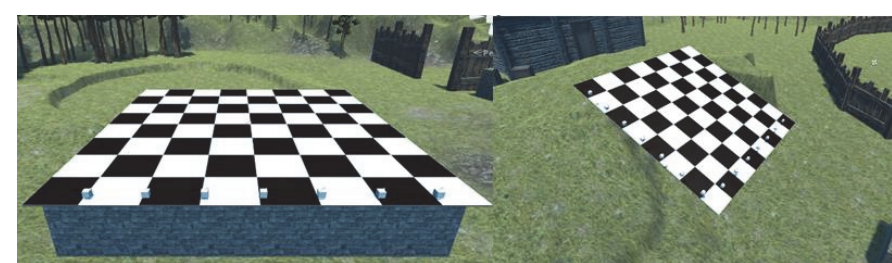

Figura 4. Se dispone de un plano horizontal (izquierda) y un plano inclinado (derecha) en donde se moverá la esfera.

Fuente: Los autores.

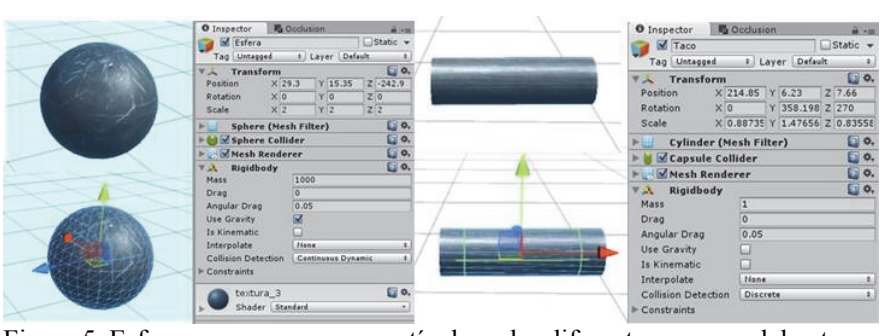

Figura 5. Esfera que se usa como partícula en las diferentes escenas del entorno inmersivo (izquierda), y el taco o impulsor de la esfera para hacer mover la esfera en una dirección determinada (derecha).

Fuente: Los autores.

un impulso. En la Fig. 3 se muestra la disposición de los elementos (esfera, taco y superficie) y la trayectoria a simular en el entorno inmersivo. Para las escenas de 1 a 4, la superficie es horizontal.

En el caso de las escenas de la 5 a 8 la esfera se ubica en la parte inferior de un plano inclinado. En la escena 5 se impulsa sobre la vertical, describiendo un mua. En la escena 6 se impulsa sobre el eje $\mathrm{x}$ describiendo un mur. En la escena 7 la esfera se impulsa en ambas direcciones, simultáneamente, lo que produce una trayectoria parabólica debido a la superposición de los movimientos de las escenas 5 y 6 . La escena 8 presenta el mismo recorrido, pero con un solo impulso diagonal sobre la esfera.

Nótese que componiendo un mua en el eje "y" con un mur en el eje " $x$ " se puede obtener un movimiento de trayectoria semi-parabólica o parabólica en el plano. Lo estratégico de usar un plano inclinado es que los estudiantes observan el movimiento más lentamente y capturen más detalles como en el caso real de una esfera de billar moviéndose sobre una superficie.

\subsection{Desarrollo del sistema de realidad virtual inmersivo}

\subsubsection{Superficie}

La creación del entorno virtual 3D para la instrucción del PSM se realizó en un paisaje rural. Las superficies (planos) cuentan con una textura cuadriculada en forma de ajedrez para facilitar la visualización del movimiento de la partícula y para que tenga una escala para referenciar el movimiento de las esferas, como se observa en la Fig. 4.

Además hay marcadores de posición en forma de cubos pequeños que se van encendiendo a medida que la esfera pasa por el punto donde está el marcador, estos cubos se han ubicado estratégicamente para dar cuenta de la velocidad y la posición

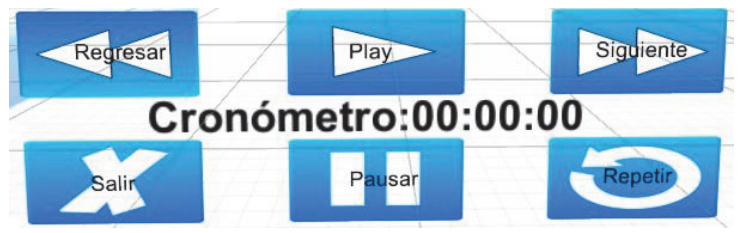

Figura 6. Menú de opciones que visualiza el estudiante dentro del entorno inmersivo

Fuente: Los autores.

de la partícula respecto al tiempo. Esto puede dar una idea intuitiva de lo rápido o lento que se mueve la partícula.

\subsubsection{Esfera e impulsor de la esfera}

Los objetos 3D son creados a partir de las formas geométricas básicas que ofrece Unity. Todos los objetos poseen una posición en el espacio (entorno de Unity) en los tres ejes $(\mathrm{x}, \mathrm{y}, \mathrm{z})$. Igualmente tiene propiedades de rotación y escalado (tamaño), lo que facilita la implementación de modelos matemáticos para simular los movimientos deseados.

La partícula es una esfera y el impulsor es un cilindro que avanza desde una posición inicial hasta colisionar con la esfera. En la Fig. 5 se pueden observar dichos objetos y sus propiedades. A los objetos se les puede agregar componentes físicos; lo que permite que sea afectado por la gravedad, colisiones con otros objetos y reciba fuerza y momento. Dicha propiedad se conoce como Rigidbody, y es utilizada en este proyecto, ya que ofrece una mayor realidad de movimiento.

\subsubsection{Menú de opciones}

Según la especificación \# 2, se debe contar con un menú de opciones, por medio del cual el estudiante tomará decisiones. Para ello, Unity ofrece una interfaz de usuario (UI) que permite crear interfaces de acceso rápido y fácil. El Canvas es el área donde se ubican los elementos UI, en este caso los botones y el texto del cronómetro es lo que tiene el estudiante para controlar el movimiento de la esfera dentro del ambiente virtual. El estudiante visualiza junto con el ambiente un menú como el que se observa en la Fig. 6.

El botón superior izquierdo permite volver a la escena anterior; con el botón superior central se impulsa la esfera; el botón superior derecho permite avanzar a la siguiente escena; el botón inferior izquierdo para salir de la aplicación; el botón inferior central para detener el tiempo del juego y el botón inferior derecho permite repetir el movimiento.

El transcurso del juego depende de las decisiones que tome el estudiante, en la Fig. 7 se encuentra el diagrama de flujo de la secuencia lógica de las escenas basado en las funciones de cada uno de los botones.

Cuando se inicia una escena se reproduce un audio guía, seguido el usuario acciona el botón play y la esfera comienza su movimiento, presentándose dos posibles situaciones: que el usuario detenga (pausa) el movimiento o que la esfera llegue a su fin. Si el tiempo del juego se detiene, las gráficas, el cronómetro y la esfera también lo harán, para continuar, se debe oprimir el botón play. Si la esfera llega al final de su recorrido, el usuario tendrá 3 nuevas opciones: repetir el movimiento, avanzar a la siguiente escena o regresar a la escena anterior. 


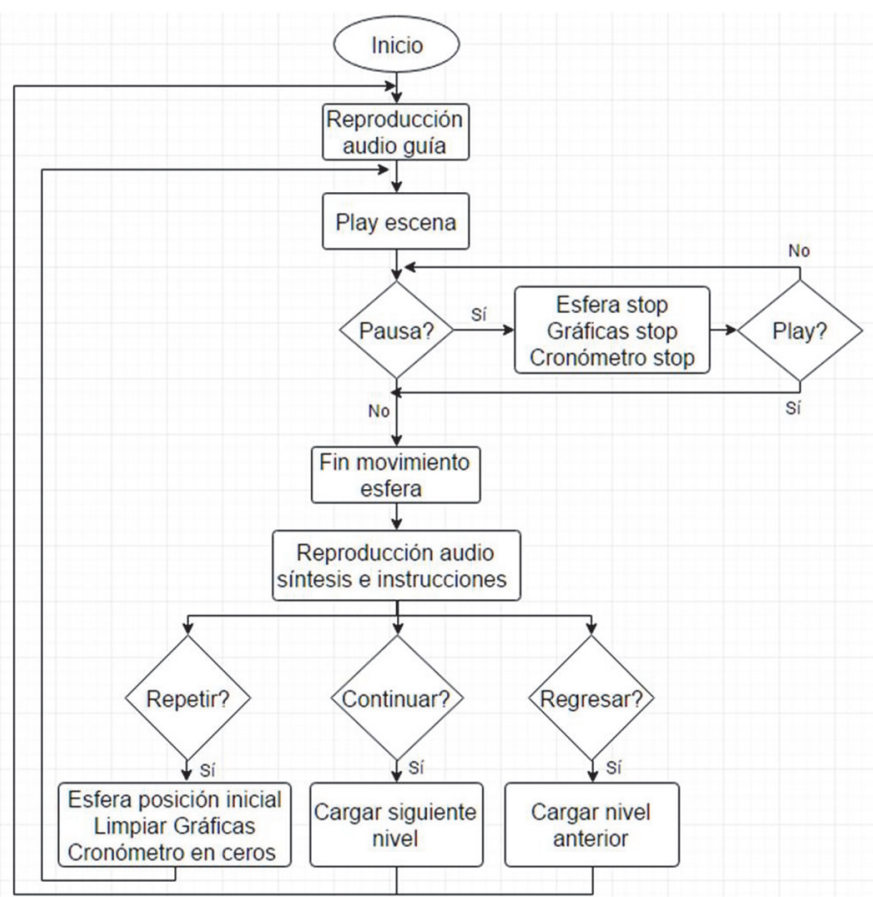

Figura 7. Diagrama de flujo secuencia lógica de las escenas en función de los botones del menú virtual

Fuente: Los autores.
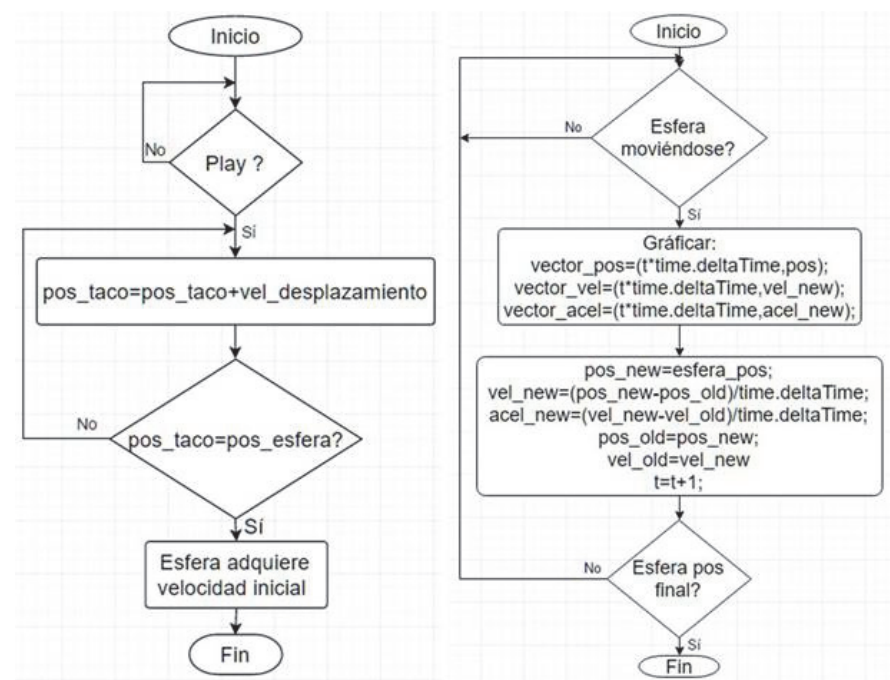

Figura 8. Diagrama de flujo de la interacción entre el impulsor y la esfera (izquierda). Diagrama de flujo de la secuencia lógica de las gráficas de movimiento (derecha).

Fuente: Los autores.

\subsubsection{Gráficas de movimiento}

Para graficar la posición, la velocidad, y la aceleración de la esfera en tiempo real, se utilizó su vector posición y el delta de tiempo (deltaTime) que ofrece Unity. En la Fig. 8 se muestra el diagrama de flujo que describe la realización de cada una de las gráficas.

Cuando la esfera adquiere movimiento, su posición en el espacio (entorno de Unity) cambia, de allí, se tiene que la velocidad será la diferencia entre la posición actual y la posición

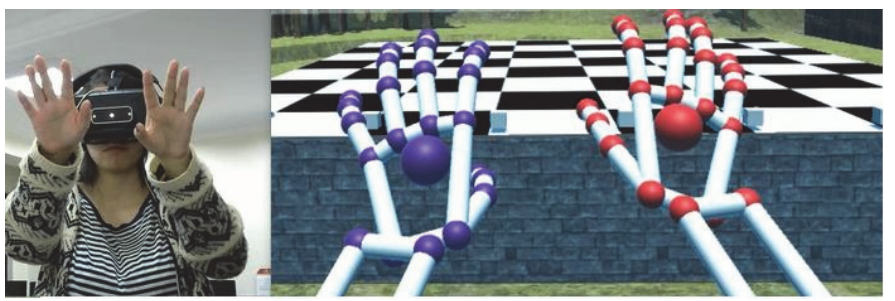

Figura 9. Prueba de funcionamiento de los dispositivos. El Leap Motion montado sobre los Oculus Rift (izquierda). Manos 3D e imagen del entorno virtual (derecha). Imagen de lo que observa el estudiante cuando entra al ambiente virtual.

Fuente: Los autores.

anterior dividida entre el delta del tiempo. La aceleración se obtiene de la resta entre la velocidad actual y la velocidad anterior, divida entre el delta del tiempo, es decir, como se calcularía numéricamente en Excel de acuerdo a las definiciones Físicas de estas variables [27].

\subsubsection{Oculus Rift y Leap Motion}

Después de haber instalado el kit de desarrollo de software (SDK) y Runtime para Windows y verificar el funcionamiento de cada uno de los dispositivos, se realiza la integración en Unity. En la Fig. 9 se muestran los dispositivos funcionando.

Cuando el estudiante en la Fig. 9 inicia la aplicación observa unas manos análogas a sus manos pero en el mundo virtual. Esto cautiva al estudiante y lo lleva a jugar, a degustar el fenómeno. Por ejemplo, el estudiante, aplaude, se coge las manos, estira los dedos, los encoge, etc. La librería de las manos 3D está programada para que cada vez que el usuario quite y ponga las manos, estas cambien de color, por eso se observan diferentes colores de manos en la Figs. 9 y 11.

\subsubsection{Efectos sonoros}

Los efectos sonoros fueron descargados gratuitamente de internet. Las instrucciones que guían al estudiante en el sistema inmersivo fueron grabadas en formato .mp4 y se reproducen acorde a las escenas y las decisiones del estudiante.

\section{Resultados}

Para alcanzar el objetivo de esta investigación, se realizó una prueba piloto con un grupo experimental para examinar que tanto apropiaron los estudiantes el PSM mediante el uso del entorno inmersivo. Se ha asumido que tal apropiación del concepto se puede representar mediante el cociente entre el cambio obtenido en los promedios de los puntajes respecto a lo que hace falta a la población por aprender. Tal cociente corresponde a un indicador de aprendizaje denominado ganancia, específicamente, la ganancia promedio normalizada, $\langle g\rangle$, de Hake de datos tomados de las respuestas de un cuestionario sobre el PSM. Participaron 20 estudiantes de los grupos de Física I (Física mecánica) de la Universidad Central, los cuales fueron sometidos a un test de selección múltiple de 9 preguntas antes y después de interactuar con el sistema inmersivo. 


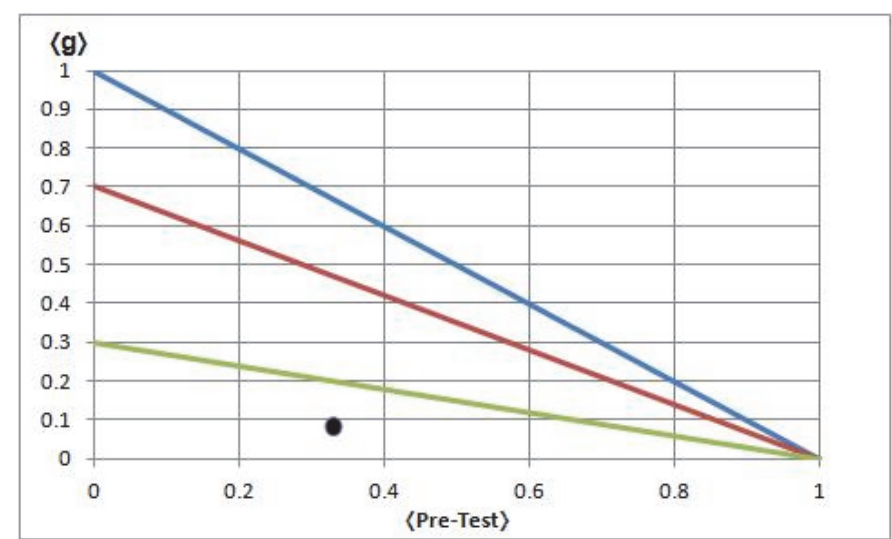

Figura 10. Se muestra la ganancia normalizada versus el promedio del pre-test; se distinguen tres regiones de niveles de ganancia: alta ganancia entre 0.7 y 1.0 , media ganancia entre 0.3 y 0.7 y baja ganancia entre 0.0 y 0.3 . En nuestro caso, el dato de ganancia normalizada cae en el rango más bajo y corresponde a un valor de $\langle g\rangle=0.08$ y una desviación estándar de la ganancia de $\Delta\langle g\rangle=0.28$. Fuente: elaboración propia

Fuente: Los autores.

Si se define que $\mathrm{P}_{\mathrm{o}}$ es el puntaje pre-test y $\mathrm{P}_{\mathrm{f}}$ es el puntaje post-test se puede calcular la ganancia normalizada de Hake [28], definida por la ec. (1)

$$
\langle g\rangle=\frac{\left\langle P_{f}\right\rangle-\left\langle P_{o}\right\rangle}{1-\left\langle P_{o}\right\rangle}
$$

Se puede construir un gráfico como el de la Fig. 10 de ganancia contra puntaje promedio de pre-test y dar cuenta de la efectividad de la instrucción con la herramienta. El resultado de la Fig. 10 indica que el sistema inmersivo para instruir el principio de superposición no generó ganancia sobre la población de 20 estudiantes intervenida. El valor de ganancia obtenido fue de $\langle g\rangle=0.08$. Este valor es menor a 0.1 y como se observa en la Fig. 10 cae dentro de la zona de baja ganancia dada entre 0 y 0.3 . Si se compara con los resultados del método con secuencia didáctica de videos usado para instruir PSM en la referencia [3] donde ellos obtuvieron $\langle g\rangle=0.29$, no se ha podido mejorar en nada la ganancia. Normalmente, el método tradicional arroja ganancias cercanas a la línea verde mientras que las metodologías activas logran ganancia entre 0.3 y 0.7 .

Aunque la muestra de estudiantes es pequeña, se puede inferir que un mundo virtual con una secuencia de escenas para instruir un tema determinado casi no origina cambios conceptuales en la población en un solo intento. Sin embargo, este resultado es poco confiable ya que la población que se intervino ya había tenido instrucción con el método tradicional y cuando se instruyó con el entorno de realidad virtual, básicamente no originó ningún cambio conceptual del PSM.

También se puede inferir que la herramienta por sí sola no es suficiente para que un estudiante mejore su comprensión de un tema determinado porque no posee acompañamiento del profesor para que lo cuestione, le refute, le haga énfasis en el conocimiento clave y le haga síntesis de lo importante.

Otro elemento relevante es que la herramienta no posee ningún momento de interacción social entre estudiantes y este ingrediente es clave para que un estudiante mejore su comprensión de un

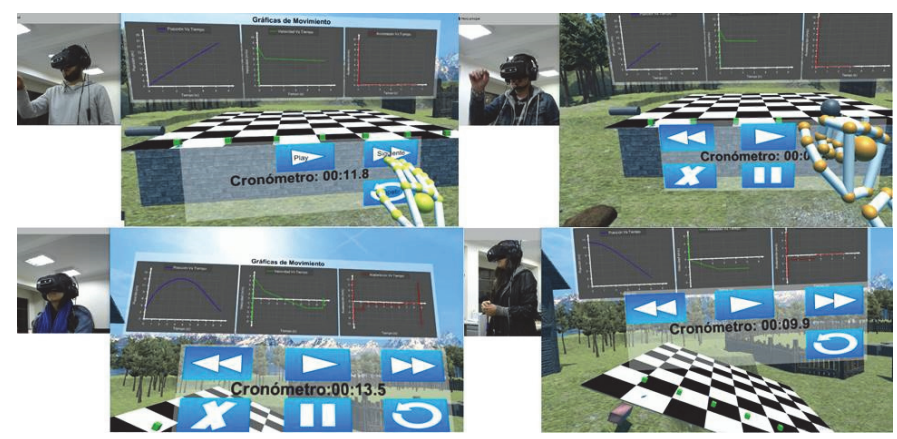

Figura 11. Interacción de algunos estudiantes en el sistema inmersivo. Las escenas se van desplegando por control del estudiante sobre un menú virtual. La secuencia se ha organizado de modo que dos escenas muestran hechos y en una tercera escena surge como resultado de las dos anteriores.

Fuente: Los autores.

determinado tema. Estos dos elementos serán vinculados en una segunda fase de investigación de la herramienta y se reportará en una siguiente publicación. Pero se debe reconocer que incorporar el elemento de acompañamiento del profesor y el elemento de interacción social entre estudiantes eleva los costos de implementación de un método de instrucción mediado como un entorno inmersivo como el diseñado en este estudio. Ahora bien, por el momento es importante no perder el norte de este estudio que solo pretende reportar el proceso de diseño, construcción y ensamble de un entorno inmersivo para instruir el PSM en estudiantes de ingeniería. Falta también, en otro momento, evaluar qué sucederá en la ganancia de una población determinada si se hacen varios intentos de uso de la herramienta. Por supuesto, no se puede dejar de lado, el bajo costo por licencia de Unity al usar un solo equipo. Si se trata de implementar masivamente la herramienta, otro elemento de costo adicional, seguramente serán las licencias del software.

El resultado clave de este artículo ha sido mostrar las etapas de diseño, construcción y puesta a punto de un entorno didáctico inmersivo para instruir el principio de superposición de movimientos. Este entorno de inmersión muestra algunas escenas como se observa en la Fig. 11. Un entorno de tal naturaleza sirve para generar un cúmulo de vivencias que el profesor puede usar posteriormente para anclar, construir lenguaje y conocimiento. Es decir, resuelve el problema de la poca experiencia que tiene el estudiante para anclar el PSM, pues como se dijo antes, el movimiento de una partícula cerca de la tierra, cuando se lanza al aire, sucede tan rápidamente que no es una experiencia adecuada para anclar el PSM.

\section{Conclusiones}

Se ha construido un entorno de inmersión 3D con audio para generar un cúmulo de vivencias, de experiencia que puede inducir al estudiante a comprender el principio de superposición de movimientos de Galileo. El entorno está constituido de un hardware descrito en la sección 2. Para su funcionamiento requiere un software que fue programado en Unity 3D. Cuando se acciona mediante la gafas de realidad virtual inmediatamente se entra en un mundo virtual que despliega un cúmulo de vivencias a través de 20 escenas que recrean una esfera moviéndose en distintas trayectorias. 
Las escenas están dispuestas en una secuencia didáctica de modo que dos trayectorias de la esfera sobre la superficie sumadas generan una trayectoria resultante. Por ejemplo, una trayectoria de mur de la esfera en el eje " $x$ " más una trayectoria de mur en el eje " $y$ " origina una trayectoria de mur de la esfera en el plano con aporte de las dos trayectorias iniciales como se ilustra en las 8 primeras escenas de la herramienta. Cada escena describe el movimiento de la partícula y además despliega en el mundo virtual la gráfica de alguna variable cinemática respecto al tiempo con el fin de que el estudiante note si cambia o se mantiene constante, esto se observa en la Fig. 11; controlado por el profesor este puede ser un elemento de gran valor pedagógico.

El entorno presenta bondades como pausar los movimientos, observar el cambio o no de variables cinemáticas de las partículas respecto al tiempo, con bastante precisión y facilidad, que difícilmente podrían verse en experimentos de laboratorios habituales.

Al probar la aplicación con un grupo de estudiantes se ha encontrado que no experimentan ganancia con el uso del entorno inmersivo, según la Fig. 10. La ganancia normalizada de Hake cae en una zona de baja ganancia, con una valor de tan solo 0,082. Este valor es casi nulo, si se compara con el método tradicional que arroja valores mayores a 0,2 o con métodos activos cercanos a 0,5 . Ya que la ganancia normalizada de Hake es un indicador estadístico que da cuenta de la efectividad de la herramienta, se asume que un entorno virtual donde el estudiante interactúa sin acompañamiento de un experto es una herramienta pedagógica incompleta. Es como si el estudiante entrara a un mundo fantástico sin un propósito determinado, quizás necesite muchas repeticiones para que produzca mejoras en la comprensión del tema que quiere aprender. Se reconoce que una población de 20 estudiantes no es representativa para calificar el entorno como inservible, pero también se reconoce que una herramienta como estas tiene faltantes y hay que adicionarle otros elementos vitales en el aprendizaje de un tema determinado como el acompañamiento de un experto y la interacción social entre estudiantes.

Un reto importante fue el diseño visual y funcional de la aplicación, ya que incluye un menú de opciones, graficas de variables cinemáticas, una superficie, una partícula y otros elementos. Se obtuvo un entorno realista y con diversas opciones, pero con información que el estudiante pasa por alto si un experto en el tema no llama la atención. A pesar de que la aplicación cuenta con grabaciones de audio que guían al estudiante, es clave la intervención del profesor para lograr claridades y anclar ideas importantes. Esto no se hizo para reconocer qué tanto alcance tiene esta tecnología, y lleva a inferir que es necesaria la acción facilitadora del profesor. Una limitación inicial para que simultáneamente no hubiese un profesor interviniendo y estudiantes con interacción entre ellos fue el costo de los equipos y dispositivos. Se hubiese requerido de al menos otros dos kits como el descrito en la sección 2.2 de la metodología y hubiese desbordado el patrocinio inicial aprobado para la investigación.

Por ser una tecnología relativamente nueva, en el ámbito educativo, las posibilidades de montar aulas con esta tecnología resultan costosas, pero lo mismo sucedió hace unos años con el proyector de video (video beam), ahora es un instrumento en la mayoría de aulas de nuestra universidad y sin duda, a largo plazo, esta tecnología de inmersión será una herramienta importante en centro educativos y universidades.

\section{Agradecimientos}

Se expresa especial agradecimiento a la Universidad Central, a la Facultad de Ingeniería y Ciencias Básicas por el apoyo en esta investigación mediante el Proyecto No 20301111 de 2016.

\section{Referencias}

[1] McDermott, L.C. and Redish. E.F., Resource letter: PER-1: Physics education research, Am. J. Phys., 67(9), pp. 755-767, 2007. DOI: 10.1119/1.19122

[2] Sánchez-Velásquez, J., Oliva-Martínez, J.M., Rosado-Barbero, L. y Cruz-González, M.I., Detección de las ideas previas en cinemática utilizando la composición de movimientos, Investig. en la Esc., 19, pp. 105-116, 1993.

[3] Barbosa, L.H. y Organista, J.O., Instrucción del principio de superposición a estudiantes de ingeniería mediante una secuencia didáctica de videos, Rev. Edu. en Ing., 9(18), pp. 106-118, 2014.

[4] Chau, M., Wong, A., Wang, M., Lai, S., Chan, K., Li, T., Chu, D., Chan, I. and Sung, W., Using 3D virtual environments to facilitate students in constructivist learning, Decis. Support Syst., 56, pp. 115-121, 2013 DOI: 10.1016/j.dss.2013.05.009

[5] Lorenzo, G., Pomares, J. and Lledó, A., Inclusion of immersive virtual learning environments and visual control systems to support the learning of students with Asperger syndrome, Comput. Educ., 62, pp. 88-101, 2013. DOI: 10.1016/j.compedu.2012.10.028

[6] Fällman, D., Backman, A. and Holmlund, K., VR in education: An introduction to multisensory constructivist learning enviroments, [En Linea]. 1999. Disponible en:

[7] http://citeseerx.ist.psu.edu/viewdoc/download;jsessionid=DE0847EFF1 5D71D69C472B536D941D54?doi=10.1.1.77.5980\&rep=rep1\&type $=p$ df

[8] Lee, E.A.-L. and Wong, K.W., A review of using virtual reality for learning, in: Transactions on Edutainment I, Springer Berlin Heidelberg, 2008, pp. 231-241. DOI: 10.1007/978-3-540-69744-2_18

[9] Flores-Cruz, J.A., Camarena-Gallardo, P. y Avalos-Villarreal, E., La realidad virtual, una tecnología innovadora aplicable al proceso de enseñanza de los estudiantes de ingeniería, Rev. Innovación Educ., 6(2), pp. 10-11, 2014.

[10] Yang, J.C., Chen, C.H. and Chang-Jeng, M., Integrating video-capture virtual reality technology into a physically interactive learning environment for English learning, Comput. Educ., 55(3), pp. 1346-1356, 2010. DOI: 10.1016/j.compedu.2010.06.005

[11] Farinzzo-Martins, V., Gregório-de Oliveira, A.J. e Guimarães, M. de Paiva, Implementação de um laboratório de realidade virtual de baixo custo: estudo de caso de montagem de um laboratório para o ensino de Matemática, Rev. Bras. Comput. Apl., 5(1), pp. 98-12, 2013. DOI: 10.5335/rbca.2013.2810

[12] Vieira-Frade, B., Campos, P. e De Sousa, P., O uso da realidade virtual como objeto de aprendizagem da matemática, Virtual Augment. Real. (SVR), 2015 XVII Symp., pp. 137-141, 2015. DOI: 10.1109/SVR.2015.27

[13] Colomeischi, A.A. and Colomeischi, T., The students emotional life and their attitude toward mathematics learning, Procedia - Soc. Behav. Sci., 180, pp. 744-750, 2015. DOI: 10.1016/j.sbspro.2015.02.192

[14] Salinas, P., González-Mendívil, E., Quintero, E., Ríos, H., Ramírez, H. and Morales, S., The development of a didactic prototype for the learning of mathematics through augmented reality, Procedia Comput. Sci. 25(81), pp. 62-70, 2013. DOI: 10.1016/j.procs.2013.11.008 
[15] Sommerauer, P. and Müller, O., Augmented reality in informal learning environments: A field experiment in a mathematics exhibition, Comput. Educ., 79, pp. 59-68, 2014. DOI: /10.1016/j.compedu.2014.07.013

[16] Boletsis, C. and McCallum, S., The table mystery: An augmented reality collaborative game for chemistry education, Lecture Notes in Computer Science, 8101, pp. 86-95, 2013. DOI: 10.1007/978-3-642-40790-1_9

[17] Merchant, Z., Goetz, E.T., Keeney-Kennicutt, W., Kwok, O.M., Cifuentes, L. and Davis, T.J., The learner characteristics, features of desktop 3D virtual reality environments. College chemistry instruction: A structural equation modeling analysis, Comput. Educ., 59(2), pp. 551568, 2012. DOI: 10.1016/j.compedu.2012.02.004

[18] Korallo, L., Foreman, N., Boyd-Davis, S., Moar, M. and Coulson, M., Do challenge, task experience or computer familiarity influence the learning of historical chronology from virtual environments in $8-9$ year old children?, Comput. Educ., 58(4), pp. 1106-1116, 2012. DOI: 10.1016/j.compedu.2011.12.011

[19] Kysela, J. and Štorková, P., Using augmented reality as a medium for teaching history and tourism, Procedia - Soc. Behav. Sci., 174, pp. 926931, 2015. DOI: 10.1016/j.sbspro.2015.01.713

[20] Jara, C.A., Candelas, F.A., Puente, S.T. and Torres, F., Hands-on experiences of undergraduate students in automatics and robotics using a virtual and remote laboratory, Comput. Educ., 57(4), pp. 2451-2461, 2011. DOI: 10.1016/j.compedu.2011.07.003

[21] Jin, G. and Nakayama, S., "Virtual reality game for safety education," in Int. Conf. Audio, Lang. Image Process. 2014, pp. 95-100.

[22] DOI: 10.1109/ICALIP.2014.7009764

[23] Lam, C.K., Sundaraj, K. and M Sulaiman,. N., Virtual reality simulator for phacoemulsification cataract surgery education and training, Procedia Comput. Sci., 18, pp. 742-748, 2013. DOI: 10.1016/j.procs.2013.05.238

[24] Lin, Y., Wang, X., Wu, F., Chen, X., Wang, C. and Shen, G., Development and validation of a surgical training simulator with haptic feedback for learning bone-sawing skill, J. Biomed. Inform., 48, pp. 122129, 2014. DOI: 10.1016/j.jbi.2013.12.010

[25] Yen, J.-C., Tsai, C.-H. and Wu, M., Augmented reality in the higher education: Students' science concept learning and academic achievement in astronomy, Procedia - Soc. Behav. Sci., 103, pp. 165-173, 2013. DOI: 10.1016/j.sbspro.2013.10.322

[26] Ibáñez, M.B., Di Serio, Á., Villarán, D. and Delgado Kloos, C., Experimenting with electromagnetism using augmented reality: Impact on flow student experience and educational effectiveness, Comput. Educ., 71, pp. 1-13, 2014. DOI: 10.1016/j.compedu.2013.09.004

[27] Chiu, J.L., Dejaegher, C.J. and Chao, J., The effects of augmented virtual science laboratories on middle school students's understanding of gas properties, Comput. Educ., 85, pp. 59-73, 2015. DOI: 10.1016/j.compedu.2015.02.007

[28] Kozhevnikov M. and Gurlitt, J. Immersive and non-immersive virtual reality system to learn relative motion concepts, in: Interdisciplinary Engineering Design Education Conference (IEDEC), 2013, pp. 168-172. DOI: 10.1109/IEDEC.2013.6526781

[29] Feynman, R.P., Leighton R.B. and Sands, M.L., The Feynman lectures on physics. Vol. I, Reading, Mass: Addison-Wesley Pub. Co., 1963.

[30] Hake, R.R., Interactive-engagement versus traditional methods: A sixthousand-student survey of mechanics test data for introductory physics courses, Am. J. Phys., 66(1), pp. 64-74, 1998. DOI: 10.1119/1.18809

D. Castro-González, estudiante de último semestre de Ingeniería Electrónica de la Universidad Central, Colombia. Experiencia de 1 año desarrollando entornos virtuales con temáticas educativas en proyectos de investigación vinculados con la Universidad Central, Colombia.

ORCID: 0000-0001-6565-9197

L.H. Barbosa, recibió la Lic. en Física de la Universidad Pedagógica Nacional, Colombia, en 1995, el título de Esp. en Ciencias Físicas en 2001, el título de MSc. en Ciencias Físicas en 2006, ambos de la Universidad Nacional de Colombia, sede Bogotá. Se graduó como Dr. en Ciencias en Física Educativa del Instituto Politécnico Nacional, México, en 2012. Ha sido docente de la Universidad Pedagógica Nacional desde el año 1998 hasta el 2004. Desde el año 2003 a la actualidad es docente de tiempo completo de la Universidad Central, Bogotá, Colombia y desde el año 2009 es catedrático de la Universidad de la Salle, Bogotá, Colombia. Fue ganador de la Presea Lázaro Cárdenas 2013, que cada año entrega el presidente de México como reconocimiento a la excelencia de quienes han contribuido a mejorar, mediante la ciencia, la vida del hombre y han dado prestigio tanto al IPN como a sus instituciones de origen. ORCID: 0000-0003-2227-6000

V. Prada-Jiménez, recibió el título de Ing. en Mecatrónica de la Universidad Militar Nueva Granada, Colombia, en 2009, el título de MSc. en Sistemas Automáticos de Producción de la Universidad Tecnológica de Pereira, Colombia, en 2012. Actualmente cursa Doctorado en Ingeniería en Sistemas Robóticos y Mecatrónicos en el Instituto Politécnico Nacional, México. Desde el 2014 a la actualidad es docente de tiempo completo del grupo de investigación MAXWELL de la Universidad Central, Colombia. Sus intereses de investigación incluyen las energías renovables, mecatrónica y bio-robótica. El profesor Prada ha sido miembro de IEEE-RAS.

ORCID: 0000-0002-4086-2989

G. Conde-Méndez, recibió el título de Ing. Electrónico de la Universidad Central, Colombia, en 2006, el título de MSc. en Ingeniería Electrónica y de Computadores de la Universidad de Los Andes, Colombia, en 2010. Actualmente cursa Doctorado en Ingeniería en la Universidad de Los Andes, Colombia. Desde el 2008 a la actualidad es docente de tiempo completo del grupo de investigación MAXWELL de la Universidad Central, Colombia. ORCID: 0000-0002-8155-5889 